\title{
Erratum to: Counting Substrate Cycles in Topologically Restricted Metabolic Networks
}

\author{
Robert D. Barish $^{(\bowtie)}$ and Akira Suyama \\ Graduate School of Arts and Sciences, University of Tokyo, \\ Meguro-ku Komaba 3-8-1, Tokyo 153-8902, Japan \\ rbarish@genta.c.u-tokyo.ac.jp
}

Erratum to:

Chapter "Counting Substrate Cycles in Topologically

Restricted Metabolic Networks" in: J. Kari et al. (Eds.),

Unveiling Dynamics and Complexity, LNCS 10307,

DOI: 10.1007/978-3-319-58741-7_14

The original version of the book was inadvertently published with the terms "undirected" and "graph" in full capitalization in Chapter 14. The erratum chapter and the book has been updated with the change.

The updated online version of this chapter can be found at http://dx.doi.org/10.1007/978-3-319-58741-7_14 